\title{
Numerical simulation of a wawe generator: a case of study.
}

\author{
Marco Trapanese, Vincenzo Franzitta, Alessia Viola \\ Dipartimento di Energia, Ing. Informazione, Mod. \\ Matematici \\ Palermo University \\ Palermo, Italy \\ marco.trapanese@,unipa.it \\ franzitta@dream.unipa.it \\ alessia.viola@unipa.it
}

\author{
Enrico Napoli, Valeria Mamola \\ Dipartimento di Ingegneria Civile, Ambientale, \\ Aerospaziale, dei Materiali \\ Palermo University \\ Palermo, Italy \\ enrico.napoli@unipa.it \\ valeria.mamola@tiscali.it
}

\begin{abstract}
The aim of present work is the numerical simulation of a linear generator, capable of directly converting the kinetic energy, available by the wave, into electrical energy, through the device linear motion (up and down).

In this paper, we intend to propose a numerical simulation approach to immersed devices by applying the Immersed Boundary Method.

The Theory of linear wave is used to study and reproduce sea conditions and the computational domain is created based on observations available for the site in which it is envisaged the positioning of the device.
\end{abstract}

Keywords-seaeave; energy; wave motion, numerical simulation

\section{INTRODUCTION}

Among the actions that the Kyoto Protocol indicates in order to reduce $\mathrm{CO}_{2}$ emissions, innovative technologies are promoted, in particular the production of electrical energy by the sea. Very important is the contribution of coastal areas in which the water can be used as driving force or exploited to its thermal and saline gradient. [1-2]

Even though the coastal areas have a great importance for energy production by waves, so far the main projects of generation systems have involved the installation in open oceans, because of the greater energy of these waves rather the coastal ones[3]. Near the coast, in fact, the waves are shorter and with a more little period so that there's a lower energy potential.

However, in closed basins such as the Mediterranean, coastal installations for wave energy recovery allow the integration of generators in coastal builds, favoring a reduction of return on investment and the decentralized production of electricity in fixed small systems.

The definition of the potential energy of optimal coastal sites is one of the basic needs for the diffusion of new technologies and for their insertions[4-6].
Wave energy depends on a lot of factors as for example the coastal distance that allows to classify the energy convertors in offshore, near to shore and shoreline devices. In areas with long coasts (Italy: $8 \mathrm{~km}$ ) the most suitable devices are the shoreline ones.

The work we intend to propose is based on the above considerations. The first step was to reconstruct the conditions of marine environment in which the device will be placed.

The Theory of linear wave is used to study and reproduce sea conditions. In this theory the hypothesis of perfect fluid (not viscous), conservative forces and irrotational motion are considered.

It was possible to use a two-dimensional domain because of the geometry of wave motion close to the coast line; there, waves tend to be parallel to the coast itself and therefore, one can study the phenomenon by referring to wavefront unit length, since the conditions are the same for the entire wavefront. Smagorinsky model is used to consider turbulence phenomena.

The second step was the device insertion within the previously created calculation domain. Finite element analysis and Immersed Boundary Method (IBM) are used to study the hydrodynamic device behavior. We analyzed a condition of firm immersed body and we obtained the extractable energy by the wave starting from the forces acting on the device faces. For energy purposes we considered only the up and down device movement due to the acting pressures on the upper and lower faces [7-8].

The first important result is the different system of thrusts to which the device is subjected when it is in correspondence of the throat or the crest of the wave. Particularly, under the throat the device is pushed upwards, under the crest it is pushed downwards.

Another very important step was the choice of the vertical velocity component value from data obtained by simulations.

Finally, we evaluated the energy through the well-known power formula.

Simulations were conducted using the software Panormus (Parallel Numerical Model for Unsteady Open-Source Flow 
Simulations), produced by the University of Palermo (Prof. E. Napoli). Panormus is a numerical tool aimed at resolving the 3D momentum equations for incompressible fluids (NavierStokes and Reynolds equations) using non-orthogonal curvilinear grids. The discretization of the equations is accomplished through the Finite-Volume method, using exahedrical cells.

So in detail, in the first section we intend to describe the wave motion and the simplifying assumptions for this application; it will also be briefly explained the problem of turbulence and numerical simulation.

The second section describes the creation of the computational domain and the wave conditions to be simulated, from observations available for the site in which it is envisaged the positioning of the device.

The third section concerns the application of IBM for the device behavior simulation in the previously simulated domain.

The last section is dedicated to the evaluation of the total thrust acting on the immersed device and the theoretical energy extractable from the sea.

\section{THE WAVES: GENERAL INFORMATIONS}

The surface layer is pushed by wind and receive from it a part of its energy that gives to the surface a higher speed than the layer below. This state involves progressively every layer with different speed that tends to drag the slower layer below and to be slowed by it [9-11]. The friction between wind and surface makes the particles of water moving with circulatory motion.

Offshore the particles of water describe almost circular orbits so at the end of every movement, they are located in a position very close to that initial one, so there's no global effective transport of material on the surface.

Shoreline, when the thickness of the water mass becomes less than half the length of the waves, the circular orbits are deformed progressively with depth till becoming elliptical near the bottom, where the particles move parallel to it with linear motion and lose most of their energy[12-14].

In a wave it is possible to distinguish two parts: the crest, the most detected and the hallow, the most depressed. Waves can be described as the result of the superposition of various sinusoidal functions of different amplitude and wavelength; however, if the height of the wave is sufficiently high compared to the depth of the seabed, we can resort to a representation based on a 'single sinusoidal function.

The simplest theory to study the phenomenon of wave motion is provided by the "linear theory" which considers the perfect fluid (not viscous), conservative forces and irrotational motion.

\section{A. Linear wave theory}

The first assumption of this is theory is the perfect fluid scheme, that is a null value of viscosity of water, so the solution of Euler equation gives an irrotational velocity field and so a potential $\phi$ linked to the velocity components[15].
The approach is based on the assumption of a condition of planar motion, this is along a direction of motion one velocity component and other two derivatives have a zero value. Because of device's position, it is possible to consider only two physical limits for the fluid: the surface and the seabed (assumed flat, impervious and horizontal).

It is possible to define a reference system whose origin is on the surface and in which along $\mathrm{x}$ direction the wave propagates and along $\mathrm{z}$ direction we measure the depth.

It is necessary to introduce specific boundary conditions to the bottom and the free surface, based on the assumptions above:

- Impervious and horizontal bottom: the vertical component of velocity $\mathrm{w}$ is null at the height $\mathrm{z}=-\mathrm{h}$, chosen as bottom level

$$
\mathrm{W}=0
$$

- Kinematic Condition for free surface: the free surface moving with liquid mass, has to be always a free surface respecting its characteristic equation in every new configuration:

$$
\frac{\mathrm{DF}}{\mathrm{Dt}}=0 \quad \text { in } \mathrm{z}=0
$$

where $F$ is characteristic equation and $\eta$ is the elevation of the wave with respect to the height $\mathrm{z}=0$

- Dynamic Condition for free surface: disregarding any spatial changes in atmospheric pressure and the effects of surface tension, the pressure wave is constant.

The conditions outlined above give rise to the expression of the free surface:

$$
-\frac{\partial \varphi}{\partial t}+\frac{1}{2} \frac{\partial^{2} \varphi}{\partial x^{2}}+\frac{1}{2} \frac{\partial^{2} \varphi}{\partial z^{2}}+g \eta=0
$$

The mathematical procedure of resolution provides a separation operation of the variables that allows the expression of the function $\Phi$ as the product of three functions, which can be calculated separately in order to achieve expression of $\Phi$ in a simpler way:

$$
\phi=\mathrm{A}(\mathrm{x}) \mathrm{B}(\mathrm{z}) \mathrm{C}(\mathrm{t})
$$

For sake of simplicity we neglect the mathematical procedure and returns the solution to the equation for each type of wave.

\section{1) Stationary wave}

Free surface:

$$
\eta=\frac{H}{2} \operatorname{coskx} \cos \left(\frac{2 \pi t}{T}\right)
$$


where:

$\mathrm{T}:$ is the period of wave

$\mathrm{H} / 2$ : is oscillation amplitude of the wave

$$
\mathrm{H}=\frac{2 \sigma \mathrm{Dcosh} k \mathrm{~h}}{\mathrm{~g}}
$$

This expression describes a standing wave whose trend of the free surface is a sine wave which does not undergo translation in the $\mathrm{x}$ direction, as the maximum and minimum of the function only vary in amplitude, as a function of time, but are always located at distances $\mathrm{L} / 2$ and $\mathrm{L}$. The potential is:

$$
\phi=\frac{\mathrm{gH}}{2 \sigma} \frac{\cosh \mathrm{k}(\mathrm{h}+\mathrm{z})}{\cosh \mathrm{kh}} \cos \mathrm{kxsen} \sigma \mathrm{t}
$$

\section{2) Progressive wave}

Free surface:

$$
\eta=\frac{H}{2} \cos (k x-\sigma t)
$$

This solution identifies wave which moves in the direction of the $\mathrm{x}$ axis with speed $\mathrm{C}=\mathrm{L} / \mathrm{T}$, where $\mathrm{L}$ is wavelength. The potential is:

$$
\phi=-\frac{\mathrm{gH}}{2 \sigma} \frac{\cosh \mathrm{k}(\mathrm{h}+\mathrm{z})}{\cosh \mathrm{kh}} \operatorname{sen}(\mathrm{kx}-\sigma \mathrm{t})
$$

where:

$\sigma:$ is the frequency of wave

$\mathrm{k}$ : is wave number

\section{B. Energy and power of a wave}

For realization of an energy converter which exploits wave motion it is essential to analyze how much energy the waves can carry and the forces acting on the device placed in the sea. In this case, we refer to a sine wave, shown in "Fig. 1":

$$
\eta(x)=\operatorname{asen}\left(\frac{2 \pi}{\lambda} x\right)
$$

By assuming the equality between kinetic energy average variation and potential energy average variation, the total energy of a surface wave, per unit of width of the wave front and for units of wavelength $\lambda$, is:

$$
\mathrm{J}_{\lambda}=\frac{2 \mathrm{~V}}{\lambda}=\frac{1}{2} \rho g \mathrm{ga}^{2}
$$

where the symbols are as shown in Fig.1.

The power of wave with unitary wave front is:

$$
\mathrm{P}=\frac{1}{4} \frac{\rho \mathrm{g}^{2} \mathrm{a}^{2} \mathrm{~T}}{2 \pi}=\frac{\rho \mathrm{g}^{2} \mathrm{~h}^{2} \mathrm{~T}}{32 \pi}
$$

If the wave interacts with the seabed loses part of its energy.

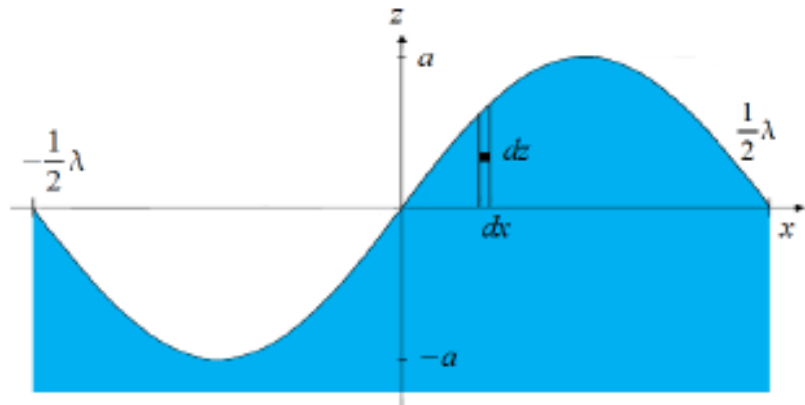

Figure 1:Sine wave

\section{RECONSTRUCTION OF WAVE CONDITIONS IN THE DOMAIN}

Since close to the coast waves are almost always parallel to the coastal line, being the conditions equal for the entire wavefront, it is possible to study the phenomenon in relation to the unit of length (of wavefront). So the domain is twodimensional and can simplify the calculations.[16]

By considering a reference system consists of a triad of Cartesian axes $\mathrm{x}, \mathrm{y}, \mathrm{z}$, the domain is as a canal which extends mainly along the $\mathrm{x}$ direction (the propagation direction of motion) and has a vertical development along the $\mathrm{z}$-direction, along which we measure the elevation of the waves. The twodimensional grid has cells:

$$
\mathrm{N}(\mathrm{x})=128 \quad \mathrm{~N}(\mathrm{y})=1 \quad \mathrm{~N}(\mathrm{z})=64
$$

After various tests, it was built a grid domain 200 meters long in $\mathrm{x}$ direction and 10 meters deep, in $\mathrm{z}$ direction, which was considered sufficient in order to reproduce the wave. The grid has this characteristics:

Along x direction:

- 32 cells of uniform width equal to 1 meter

- 8 cells of decreasing width

- 16 cells of uniform width equal to 1 meter ( coating 35.5$36.5 \mathrm{~m}$ distance where will be added the device)

- 8 cells of increasing width

- 32 cells of uniform width equal to 1 meter

- 32 larger cells of increasing width.

Along z direction:

- 64 cells of decreasing size from the bottom to the free surface.

Condensation of the meshes in the central cells of the domino, not necessary at this stage, was realized so preparatory to the second step of simulation, in anticipation of positioning the generator, to ensure greater accuracy of the interpolations.

The computational domain requires appropriate boundary conditions to ensure the coupling between the wave field on 
the computational grid and the field in the infinite domain, left out of the calculation. All the waves are outgoing and must be absorbed on the open boundaries, otherwise reflect, impairing the accuracy of the results[17].

The perfect absorption can be achieved only in specific cases, but in general, it has been shown that artificial damping can be achieved by adding a "sponge layer" that absorbs the outgoing waves.[9]

In the present case, the "sponge layer" is formed from the cells next to the outflow domain, characterized by a greater size of the wavelength $(\mathrm{L} \approx 25 \mathrm{~m}$ ).

A particular of the 'thickening' of grid is shown in Fig.2.

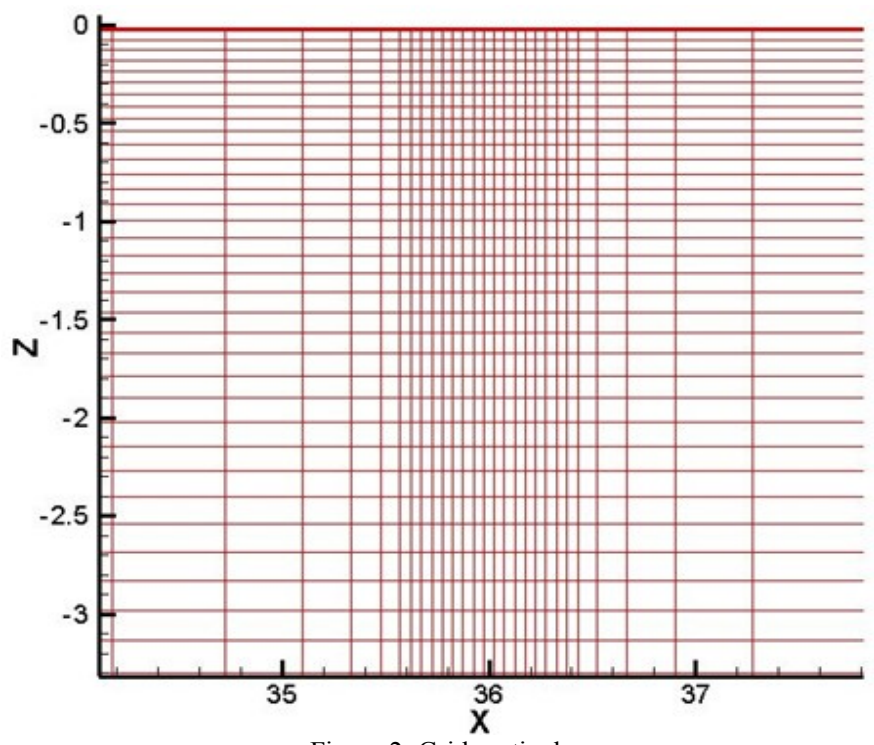

Figure 2: Grid particular

The next step was to define the boundary conditions.

Inlet section: introduction of a condition on the height $h$ at the entrance, type "time-dipendent", to reproduce the actual conditions of the surface, known from the available observations on the area of Trapani. The sinusoidal law which expresses the trend of the free surface is defined as follows:

$$
h=\operatorname{Hsen}\left(\frac{2 \pi t}{T}\right)
$$

Outlet section: hydrostatic distribution of pressure and null derivatives of the considered variables.

Lateral boundaries: void derived variables of the turbulence model

Upper boundary: hydrostatic distribution of pressure and null derivatives of the considered variables.

Lower boundary: logarithmic law of velocity.

The last condition follows from the assumption that the turbulent motion can be considered as formed of many particles dispersed in the fluid, as a kind of gas, between which do not occur elastic collisions, but the momentum is transferred to mixing when the particle encounters those surrounding in its movement in a direction transverse to the current.[18]

According on Linear wave theory, we report the expressions of free surface and velocity components used to reconstruct sea conditions:

$$
\begin{aligned}
& \eta=\frac{H}{2} \cos k x \cos \left(\frac{2 \pi t}{T}\right) \\
& \mathrm{u}=\frac{\mathrm{gHk}}{2 \sigma} \frac{\cosh \mathrm{k}(\mathrm{h}+\mathrm{z})}{\cosh \mathrm{kh}} \cos (\mathrm{kx}-\sigma \mathrm{t}) \\
& \mathrm{w}=\frac{\mathrm{gHk}}{2 \sigma} \frac{\operatorname{senh} \mathrm{k}(\mathrm{h}+\mathrm{z})}{\cosh \mathrm{kh}} \operatorname{sen}(\mathrm{kx}-\sigma \mathrm{t})
\end{aligned}
$$

where symbols have the meaning as given before and the height is measured between crest and trough.

The first simulation was made with a $\mathrm{H}=1 \mathrm{~cm}$ to validate the approximation of Linear wave, that is valid for small height waves and verify that the constructed domain was good.

Results were satisfactory so we made simulations with real magnitudes of motion, that is $\mathrm{H}=1 \mathrm{~m}$ and $\mathrm{T}=4 \mathrm{~s}$ and it was run for 150 seconds.

During simulations, the grid was periodically regenerated along vertical direction with an upper-end condition, that is height of the cells growing along the $\mathrm{z}$ direction.

In Fig.3 there's an example of regenerate grid during the simulation.

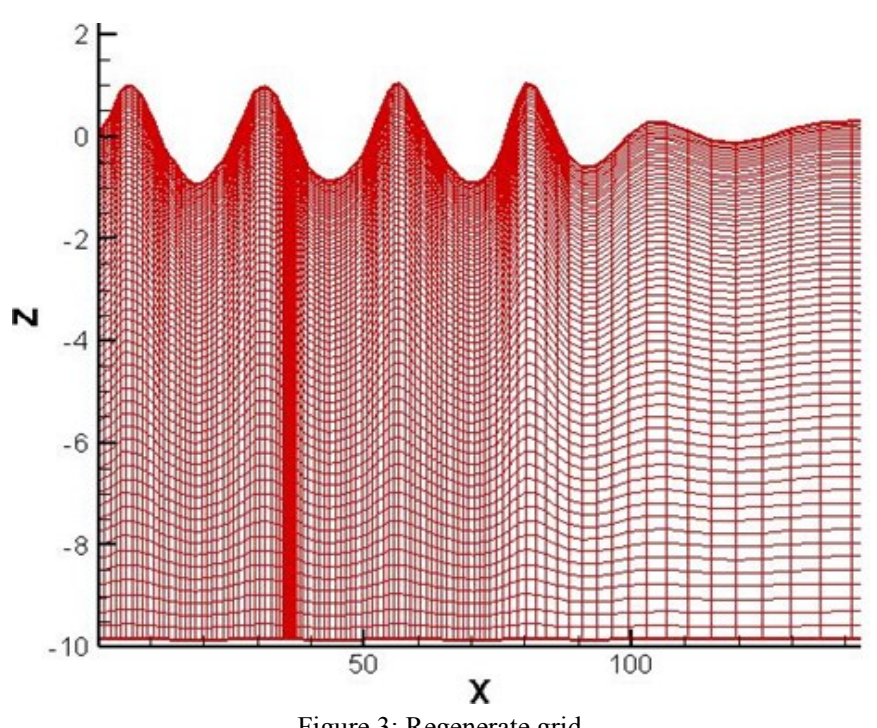

Figure 3: Regenerate grid

The process phenomenon is part of the macro argument "Turbulence".

In the case of turbulent flow fields, time and space oscillation frequency it is very high and then it is difficult to completely describe the evolution of the velocity.

The simplest approach to overcome this problem is a statistical modeling of turbulence. 
This involves using a model to filter all the unpredictable fluctuations of flow around its "average performance". The turbulence is characterized by disordered motion that result in random fluctuations in time and space.

A generic periodic function can be expressed as the sum of infinite periodic sinusoidal functions, of different size. This can be extended in random functions: in this way a field of a turbulent flow can be represented as a superposition of several oscillations of different amplitude and frequency.

At the macroscopic level, the turbulence on the mean flow occurs through the generation of large vortices: the inertial forces are dominant then create an oscillating motion, which produces more instability and fluctuations of decreasing amplitude.

The wave motion is a phenomenon in which the turbulent efforts are limited and therefore it was considered more suitable the use of an approach to LES (Large Eddy Simulation), which solves directly the larger structures of the turbulence and model only smaller ones. Since turbolence is not to developed, there's the advantage of simplifying the grid construction, that is the main problem when the field of turbulent motion is very oscillating [19].

\section{IMMERSED BOUNDARY METHOD}

In computational fluid dynamics, the immersed boundary method is an approach to simulate fluid-structure interactions. In general it consist in three main phases

1. Identification of solid-fluid interface as a discontinuity: the outline $\psi$ represented by interfacial markers $\left(i_{b}\right)$

2. Defining a grid-interface. For each marker point $\left(i_{b}\right),(a t$ this stage it is not yet known what are fluid or solid points) neighboring points $\mathrm{X}(\mathrm{i}, \mathrm{j})$ of the Cartesian grid on both sides of the interface are identifyied: the system is solved for each point in the vicinity of interface using the method of Newton-Raphson. By appropriate calculations are identified solid and the fluid points.

3. Evaluation and distribution of the forcing function in correspondence of the points: each node is classified as solid point $\mathrm{I}_{\mathrm{B}}$ if it lies in the fluid phase.

The point $\mathrm{I}_{\mathrm{P}}$ is obtained as the intersection between the $l$ line (passing through $\mathrm{I}_{\mathrm{B}}$ and perpendicular to the surface) and the surface itself. The $\mathrm{P}_{\mathrm{P}}$ is identified on the opposite side of $l$ line with respect to the point $I_{B}$, by requiring that the distance $d_{2}$ between $P_{P}$ and $I_{B}$ is equal to the distance $d_{1}$ between $I_{P}$ and $I_{B}$. After identifying the points $I_{P}$ and $P_{P}$, the velocity at the point of the current $I_{B}$ can be obtained by linear interpolation between the velocities $U_{I P}$ and $U_{P P}$. Since the $I_{P}$ points lie on immersed boundary, the speed $U_{I P}$ coincides with immersed surface's one, which is known.[20] The speed $U_{P P}$ can be obtained through interpolation of the speed of the surrounding nodes, using a suitable technique to grids curvilinear. This procedure is described in "Fig. 4".

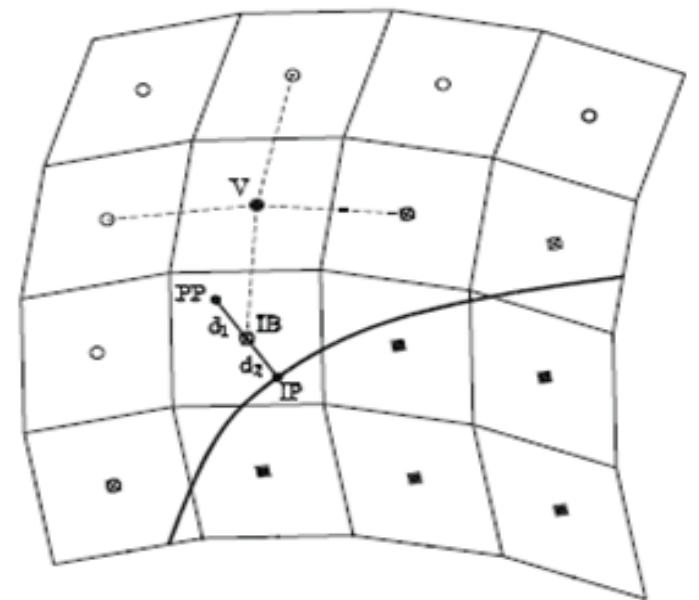

Figure 4: Identification of solid fluid and immersed boundary nodes, in a twodimensional grid

To carry out the simulation of Immersed boundary in the above described domain, the device is considered as a cylinder of dimensions:

$$
\begin{aligned}
\text { Height } & =1 \mathrm{~m} \\
\text { Diameter } & =0.7 \mathrm{~m}
\end{aligned}
$$

and covers, along $\mathrm{z}$, the heights

$$
z=(-2,5)-(-1,5) m
$$

and along $\mathrm{x}$ the distance

$$
\mathrm{x}=35.65 \text { to } 36.35 \mathrm{~m}
$$

Otherwise simulation performed for the wave motion, a filter is introduced in the regeneration vertical grid to eliminate improper oscillations of the free surface and prevent the onset of instability. The first simulation made with these conditions had a positive outcome and to ensure the accuracy of the results other tests were carried out with a greater number of iterations and smaller time intervals. The simulation was conducted, again, for a period of $150 \mathrm{~s}$.

\section{A. Device under the hollow of a wave}

When the device is located in the trough of the wave, the horizontal velocity component " $u$ " assumes negative values all around the device except the region next to the upper face, where horizontal velocity component assumes positive values.

On the contrary, vertical velocity component " $w "$ has positive values all around the lower face, except in a small area almost close to the surface, in which values are slightly negative; in the upper zone speeds are greater in absolute value, but predominantly negative. These results are reported in "Fig. 5-6" that are images reproduced by the software during the simulation. 

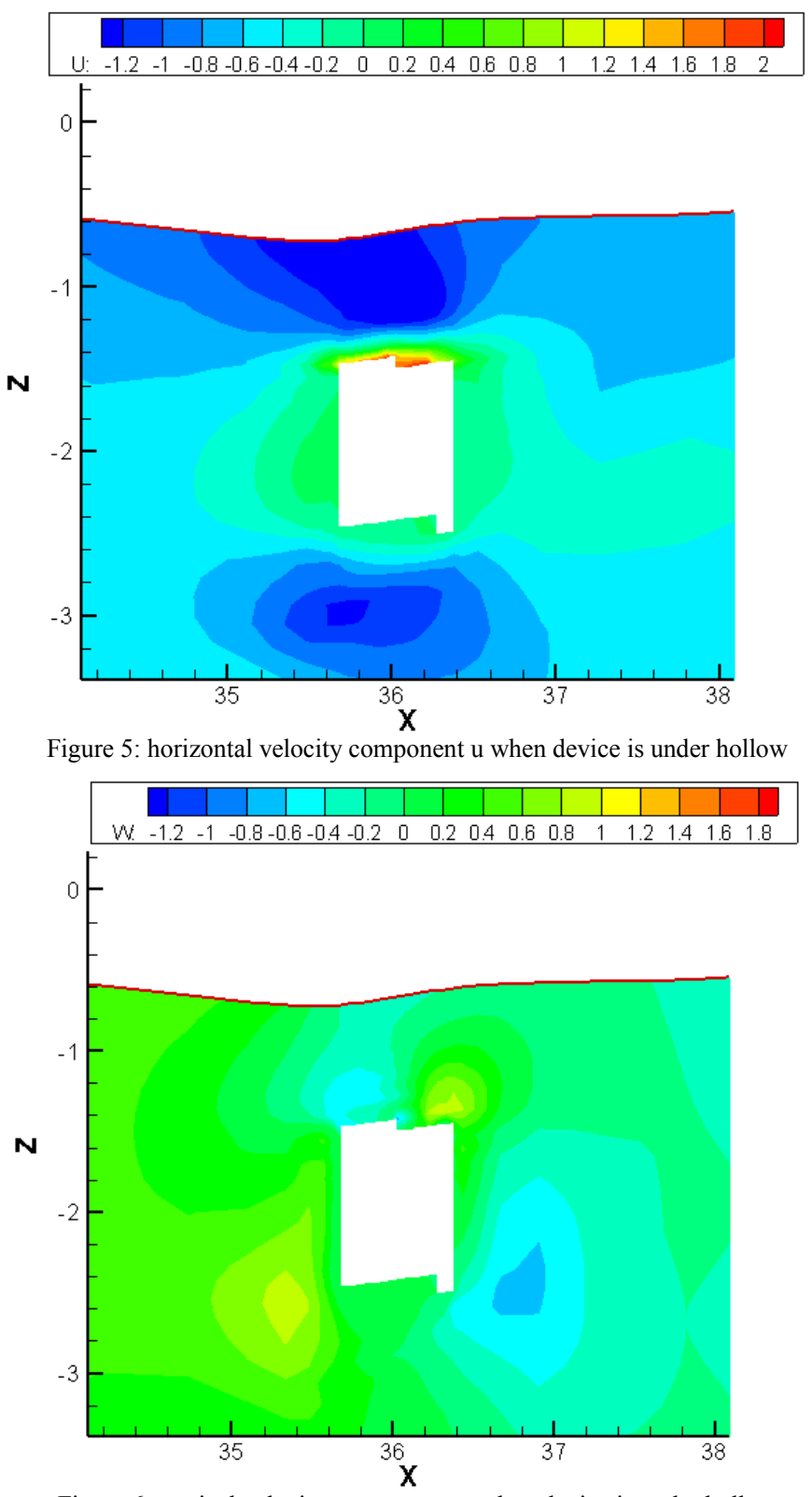

Figure 6: vertical velocity component $\mathrm{w}$ when device is under hollow

\section{B. Device under the crest of a wave}

In the area next to the upper face, " $u$ " is always negative and increases with altitude until reaching very high values. In the lower zone is always positive with increasing values downward. The vertical component $w$ assumes positive values in the lower part, while the area next to the upper face is characterized by negative $" w$ " and positive values are found away from it, as shown in Fig.7-8.

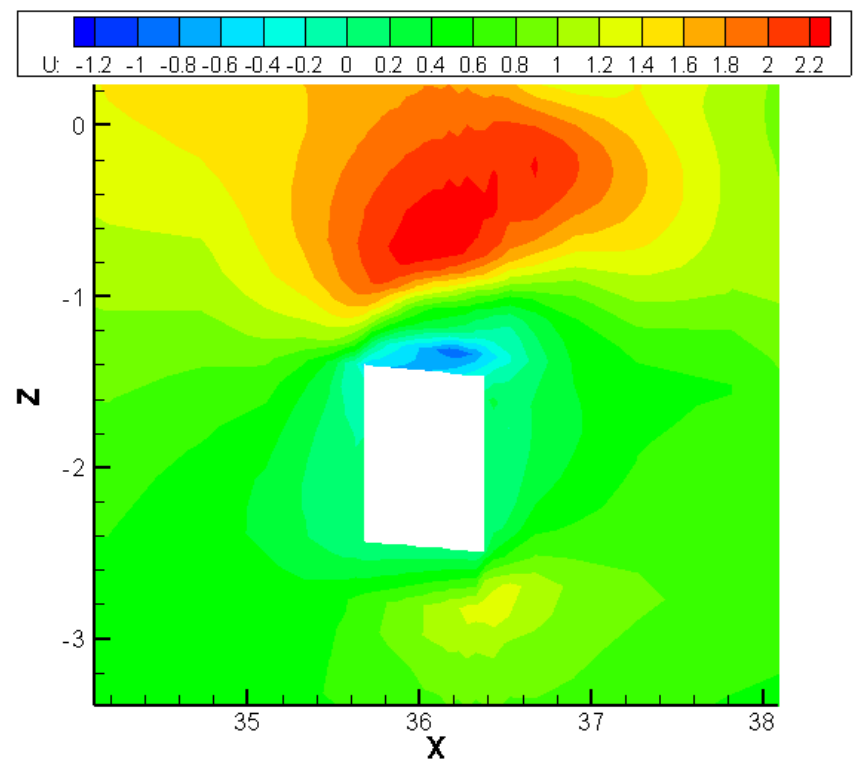

Figure 7: horizontal velocity component $\mathrm{u}$ when device is under crest

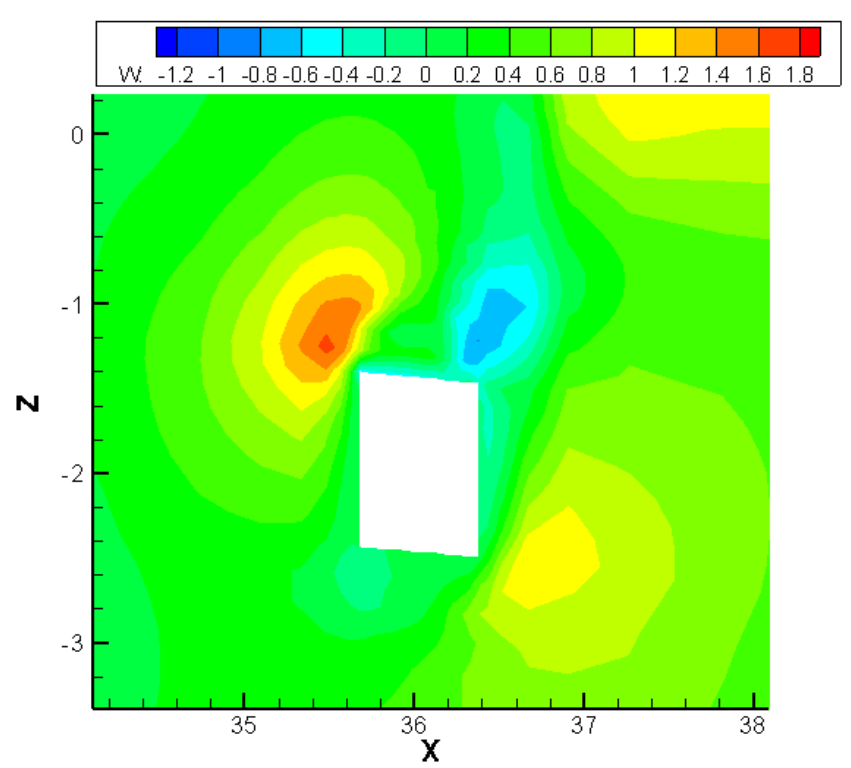

Figure 8: vertical velocity component w when device is under crest

\section{THEORETICAL ENERGY EXTRACTABLE FROM THE SEA}

Found that the model reproduced optimally sea conditions and immersed body behavior, the next step was to evaluate the thrust that the device receives, that has variable direction: under the crest pushing agent is down, under the hallow pushing agent is upward. This result can be explained by Bernoulli's theorem:

$$
\mathrm{p}_{\text {down }}-\mathrm{p}_{\text {up }}=\frac{1}{2} \rho \mathrm{v}_{\text {up }}^{2}-\frac{1}{2} \rho \mathrm{v}_{\text {down }}^{2}
$$

When the device is below the wave crest, the current lines are directed along the direction of wave motion and are concentrated below the device itself (the motion goes from 
higher pressure zones to lower pressure zones) as in "Fig 9"; in this condition the speed is positive close to the lower face and negative close to the upper face, so pressures will be positive on the upper side and negative on the lower one: the result is that the generator is pushed downwards.

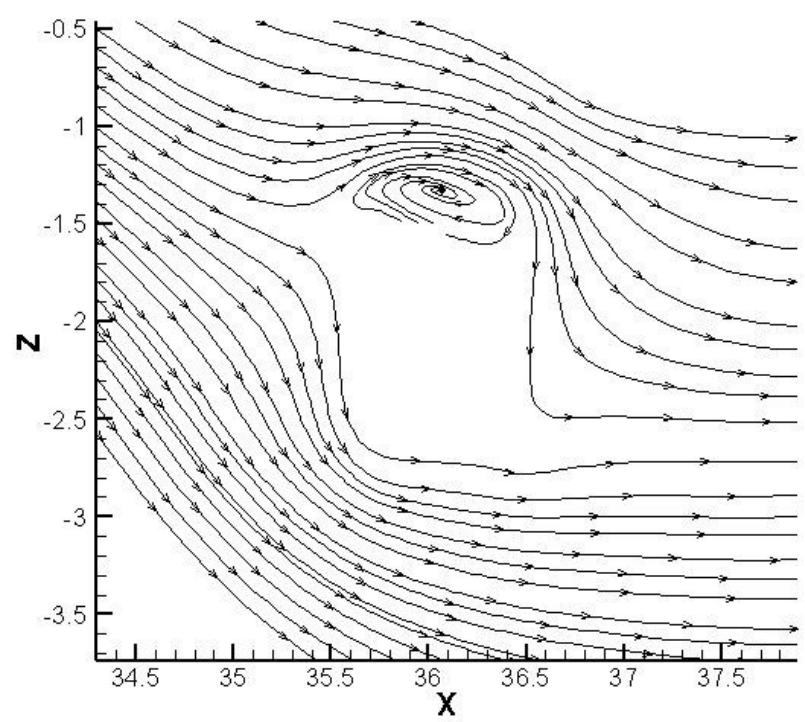

Figure 9: Current lines below a crest

An opposite situation is when the device is under the hallows where the wave velocity is negative and the current lines are oppositely directed relative to the direction in which the waves propagate (Fig.10), but close to the upper face the values of speed are positive and greater in absolute value than the lower face. A contrary situation occurs as regards the values of the pressure and that is, the prevailing pressure is on the lower face then the overall thrust is pointing upwards . (Figure 45).

The calculation of the thrusts was conducted on the basis of the values of the pressure acting on the lower and upper face calculated instant by instant, along the course of the simulation using the software Panormus. These performance measures are shown in the Fig.11-12-13.
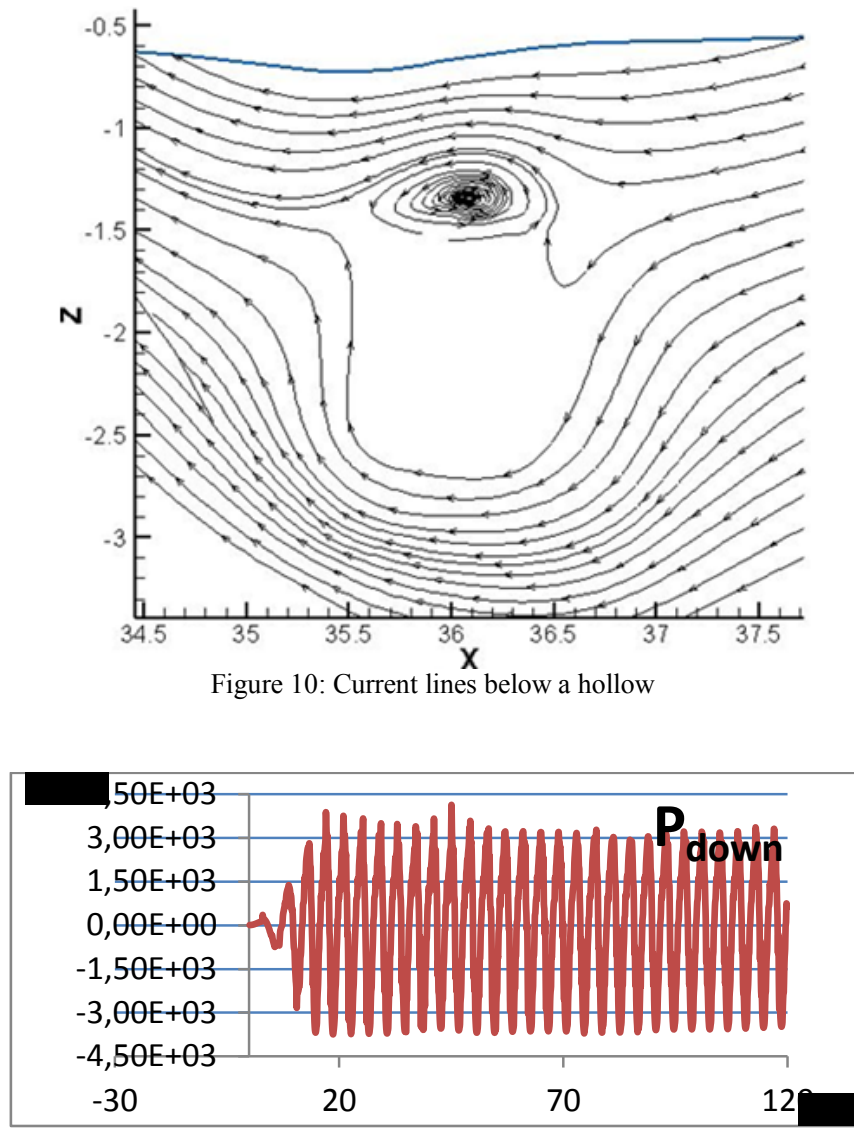

Figure 11: temporal trend of pressure on lower face

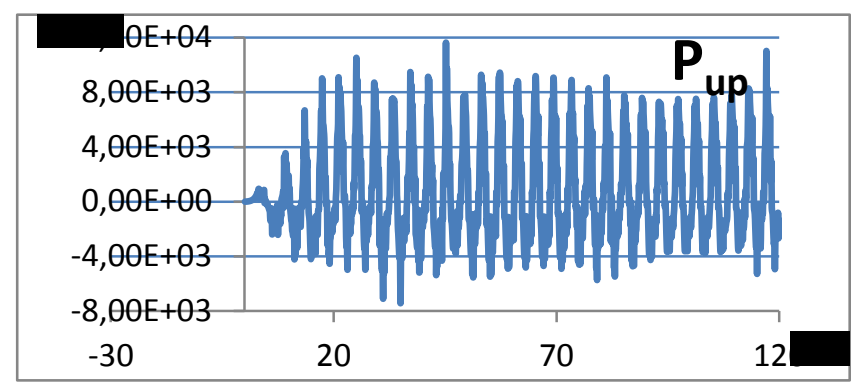

Figure 12: temporal trend of pressure on upper face

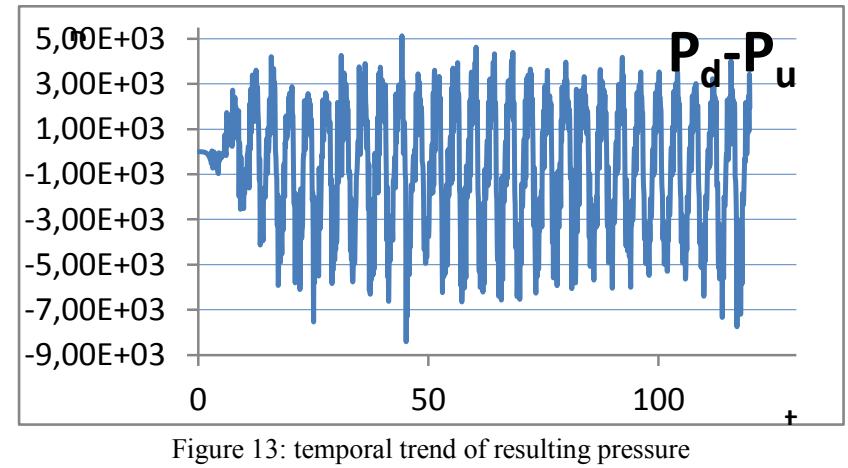

The generator has been considered in this phase as a stationary body, so that it was possible to evaluate the forces on it agent. 
A more detailed evaluation would require that the body was free to move in order to evaluate the displacements due to the boost received, but this is not the subject of this study.

What can be evaluated in this work is the power theoretically obtainable from the device considering the action of the pressure, referring to a deepening of the electric type to take into account the mode of generation and energy efficiency.

By observing the change in time of the pressure difference $\Delta \mathrm{p}$ between lower and upper face, a value of about 10000 $\mathrm{N} / \mathrm{m}^{2}$ was chosen.[1-3]

Given the two-dimensional geometry of the problem, it was possible to calculate the energy extractable from the sea per width unit of the wavefront; in fact, by multiplying the value of $\Delta \mathrm{p}=10000 \mathrm{~N} / \mathrm{m}^{2}$ by the device diameter $(\mathrm{D}=0.7 \mathrm{~m})$, we got an approximate value of force per width unit of wavefront equal to $\mathrm{F}=7000 \mathrm{~N} / \mathrm{m}$.

Recalling that the power, in the case of constant force, is defined as the derivative of the work (in this case the work is done by the difference of pressure per linear meter) over time:

$$
P=\frac{d L}{d T}
$$

that is

$$
\mathrm{P}=\mathrm{F} \cdot \mathrm{W}
$$

\section{$\mathrm{F}=7000 \mathrm{~N} / \mathrm{m}$}

$\mathrm{w}=$ is the vertical velocity component that is estimated approximately equal to $0.6 \mathrm{~m} / \mathrm{s}$ on the basis of simulation result analysis.

However, recalling the Clapeyron's theorem, which states that "the work of external forces is equal to twice the energy of deformation which measures the work produced to bring the body from the reference configuration to the current one", considering a value of force equal to half of $\mathrm{F}$ :

$\mathrm{F}^{\prime}=3500 \mathrm{~N} / \mathrm{m}$.

The observations made above, allow an estimated value of theoretical power equal to: $\mathrm{P}=2100 \mathrm{~W} / \mathrm{m}=2,1 \mathrm{KW} / \mathrm{m}$.

To obtain a result in terms of energy per unit length can be very useful in order to compare the power available from traditional technologies, or better yet, to evaluate the configuration in the sea corresponding to a target value of power.

With the availability of data on the characteristic quantities of the wave, you can estimate the power per meter owned by the wave:

$$
P=\frac{\rho g^{2} h^{2} T}{32 \pi}
$$

$\rho=$ density

$g=$ gravity acceleration

$h=$ height measured between the crest and the trough $[2 \mathrm{~m}]$

$\mathrm{T}=$ wave period $[4 \mathrm{~s}]$

Substituting numerical values, we obtain a power approximately $16 \mathrm{~kW} / \mathrm{m}$.
This large difference between the energy possessed by the wave and the energy extracted by the device, in the particular configuration studied, if on the one hand show that the recovery efficiency is not high, on the other hand, leaves ample space for improvement. In fact, the simulations conducted for the purpose of the present work, put more attention on the reproduction of the conditions of wave motion and behavior of the immersed body, but leave open the way for new studies to maximize the performance of the device, such as varying the position. The results therefore show that the production of electricity from the sea, although is a sector that is still largely unexplored, it offers great potential energy which, when properly managed, can provide performance comparable to those provided by the technologies already established in the energy market.

\section{ACKNOWLEDGment}

This work was funded by Ministero dell'Ambiente e del Mare through IMPETUS project.

\section{REFERENCES}

[1] P. Holmes .'(2001): "Coastal processes: Waves"; ${ }^{1}$ Department of Civil and Environmental Engineering Imperial College, England

[2] F. Carli, S. Bonamano, M. Marcelli, M.A. Peviani: "Metodologia per la valutazione del potenziale energetico associato al moto ondoso in acque costiere".

[3] D. Vicinanza, Dipartimento di Ingegneria Civile, Seconda Università degli Studi di Napoli. "Potenzialità dell'energia ondosa lungo le coste italiane".

[4] E. Balaras(2004): "Modelling complex boundaries using an external force field on fixed Cartesian grids in large-eddy simulations". Comput Fluids 33: 375-404

[5] R. Mittal, G. Iaccarino (2005): "Immersed Boundary Methods". Annu. Rev. Fluid Mech. 37:239-61

[6] Napoli E. (2010): Panormus User's Manual. Dipartimento di ingegneria civile, ambientale e aerospaziale, Università degli studi di Palermo.

[7] Roman F., Napoli E., Milici B., Armenio V. (2009): "An improved immersed boundary method for curvilinear grids". Computers and Fliuds 38:1510-1527.

[8] Mittal R.,Iaccarino G. (2005): "Immersed Boundary Methods". Annu. Rev. Fluid Mech. 37:239-61

[9] Israeli M., Orszag S.A., (1981): "Approximation of Radiation Boundary Conditions". J. Comput Phys., 41: 115--131.

[10] Bonanno A., Franzitta V., Muzio F.P., Trapanese M., "A multiphysics approach to the design of a seawave energy conversion system", Proceedings of ICSET, Singapore, ,p.p. 665-668,art. no. 4747090, 2008.

[11] Franzitta V., Rizzo G., "Renewable energy sources: A mediterranean perspective”, Proceedings ICBEE 2010-2nd International Conference on Chemical, Biological and Environmental Engineering, , p.48-51, art. no. $5652332(2010)$.

[12] Di Dio V., Franzitta V., Muzio F., Scaccianoce G., Trapanese M., "The use of sea waves for generation of electrical energy and hydrogen". MTS/IEEE Biloxi - Marine Technology for Our Future: Global and Local Challenges, OCEANS, 2009, art. no. 5422319.

[13] Sorrentino G., Scaccianoce, G., Morale, M., Franzitta, V., "The importance of reliable climatic data in the energy evaluation", Energy Volume 48, Issue 1, pp74-79, December 2012.

[14] Trapanese M., Viola A., Franzitta V., "Description of hysteresis of nickel metal hybride battery". IECON 2012, 38th Annual Conference on IEEE Industrial Electronics Society, Montreal, Canada, pp. 967-970. 
[15] Franzitta V., Viola A., Trapanese M., "Description of hysteresis in Lithium battery by classical Preisach model", Advanced Materials Research Vols. 622-623, pp 1099-1103, 2013.

[16] Ciulla G., Franzitta V., Lo Brano V., Viola A., Trapanese M., "Mini Wind Plant to Power Telecommunication Systems: a Case Study in Sicily", Advanced Materials Research Vols. 622-623 pp 1078-1083, 2013.

[17] Trapanese M., "A model of a linear synchronous motor based on distribution theory", J App Phys, vol.111 ,Article number07E731, 1 April 2012.

[18] Trapanese M, "Noise enhanced stability in magnetic systems", J. App. PhysVolume 105, Issue 7, Article number 07D313, 2009.

[19] Cirrincione M., Miceli R., Galluzzo G.R., M. Trapanese,” Preisach function identification by neural networks", IEEE Transactions on Magnetics Volume 38, Issue 5 I,pp 2421-2423, 2002.

[20] Trapanese M.; Franzitta V., Viola A. "The Jiles Atherton Model for Description Of Hysteresis in Lithium Battery" Conference Proceedings - IEEE Applied Power Electronics Conference and Exposition - APEC 2013- Long Beach,(CA), March 2013-978-1-4673-4355-8, pp 27732775. 